\section{Inequidades de acceso a la información e inequidades en salud}

\author{
Alberto Pellegrini Filho ${ }^{1}$
}

Palabras clave: acceso a la información, Biblioteca Virtual de Salud, inequidad, nuevas tecnologías de información y comunicación, salud.

\footnotetext{
Coordinación de Investigaciones, División de Salud y Desarrollo Humano (HDP/HDR), Organización Panamericana de la Salud. La correspondencia debe enviarse a Alberto Pellegrini-Filho, PAHO/ WHO (HDP/HDR), 525, 23rd St., NW, Washington, D.C. 20037, Estados Unidos de América. Correo electrónico: pellegri@paho.org
}

En este artículo buscaremos presentar evidencias de que las inequidades de información son un importante determinante de las inequidades en salud y de que la superación de dichas inequidades de acceso a la información, particularmente a través del uso de las nuevas tecnologías de información y comunicación (TIC), puede significar un importante avance en el sentido de garantizar a todos el derecho a la salud.

En los últimos años se ha observado un gran crecimiento de la literatura científica internacional dedicada al estudio de las inequidades en salud y sus determinantes $(1,2)$. Dos son los hallazgos más significativos de esos estudios. El primero es la constatación de que, una vez superado un determinado límite de crecimiento económico de un país (cerca de 8000 a 10000 dólares estadounidenses -USD - per cápita), un crecimiento adicional no agrega mucho en términos de mejoría de las condiciones de salud. A partir de ese nivel, el factor más importante para explicar la situación general de salud de un país no es su riqueza total, sino la manera como se distribuye esa riqueza (3). El segundo es haber identificado el deterioro del llamado capital social, o sea, de las relaciones de solidaridad y confianza entre personas y grupos, como un importante mecanismo a través del cual las inequidades de renta repercuten negativamente sobre la situación de salud. En otras palabras, el deterioro del capital social en sociedades inequitativas sería un importante factor para explicar por qué su situación de salud es inferior a la de sociedades con relaciones de solidariedad más desarrolladas. Las inequidades socioeconómicas menoscaban la cohesión social y conllevan una menor participación política. Países y estados con grandes inequidades de renta, escasos niveles de cohesión social y baja participación política son los que menos invierten en capital humano y en redes de apoyo social $(4,5)$.

Los países de América Latina y el Caribe (ALC) tienen una carga doble: además de presentar graves inequidades en la distribución de la riqueza, tienen grandes sectores de población que viven en condiciones de pobreza. Recientemente, el tema de la pobreza también viene llamando la atención de muchos autores, generando un cambio en la manera como la entendemos y en las formas de combatirla. Para Amartya Sen, premio Nobel de economía, pobreza no es solamente la falta de acceso a bienes materiales, sino también la falta de oportuni- 
dades y de posibilidades de opción entre diferentes alternativas. El Banco Mundial agrega también a su definición de pobreza la falta de voz frente a las instituciones del Estado y de la sociedad y la vulnerabilidad frente a imprevistos; en su último informe (2000-2001) recomienda que el combate a la pobreza debe incluir tanto la generación de oportunidades económicas como medidas que favorezcan la construcción de redes de apoyo y una mayor participación de los pobres en la vida social (6).

La promoción del empoderamiento y el fortalecimiento del capital social vienen siendo consideradas como estrategias fundamentales para superar las desigualdades y la pobreza y, en consecuencia, mejorar la situación de salud, particularmente de los pobres o excluidos. Las TIC pueden contribuir de forma importante al desarrollo de estas estrategias, a través de diversos mecanismos. En primer lugar, por su capacidad de influir sobre importantes determinantes generales de la salud, como las relaciones sociales, los procesos culturales y otros aspectos fuertemente relacionados con el bienestar físico, mental y social. En segundo lugar, las nuevas TIC están redefiniendo el proprio concepto de comunidad, liberándola de los límites geográficos. Las personas pueden estar hoy en constante contacto con sus familiares, amigos y colegas en todo el mundo, utilizando buscas, teléfonos celulares, correo electrónico, etc. Estas comunidades virtuales no deben ser despreciadas como menos reales que las otras; están cambiando la manera como entendemos la creación y mantenimiento del capital social y pueden ofrecer importante apoyo, particularmente para los que están aislados por la edad o defectos físicos o para los que viven en lugares muy lejanos. En tercer lugar, las TIC tienen un importante impacto sobre las inequidades en salud, en la medida en que permiten ampliar el acceso a la información por parte de grupos excluidos de los procesos de toma de decisiones de cualquier tipo, trayendo como consecuencia el empoderamiento de esos grupos, su constitución como actores sociales $\mathrm{y}$, en ultima instancia, el fortalecimiento del proprio proceso democrático. Las TIC permiten a esos grupos aumentar su conocimiento de los problemas locales y globales, estrechar sus relaciones con otros grupos y fortalecer su organización e inserción en acciones colectivas.

La Biblioteca Virtual de Salud (BVS), coordinada por BIREME/OPS (Organización Panamericana de la Salud), busca aprovechar el potencial de las TIC para democratizar la información y el conocimiento, favoreciendo una participación social más amplia en la formulación de políticas volcadas a la promoción de la equidad en salud. Además de una colección organizada de fuentes de información en formato digital con capacidad de almacenaje infinita y acceso universal en tiempo real, la BVS es también una plataforma virtual que permite interacciones entre productores, intermediarios y usuarios del conocimiento.

Sin embargo, todo este potencial de la BVS está amenazado de no realizarse si no se resuelve un problema fundamental, la chamada "brecha digital", más impresionante que cualquier otra inequidad y con un enorme poder para generar y ampliar otras inequidades. De los casi 513 millones de usuarios de Internet estimados para el año 2001, el 35\% vivía en Estados Unidos (EE. UU.) y Canadá, el $30 \%$ en Europa, el $28 \%$ en Asia y el $5 \%$ en ALC (cuadro 1).

Según un estudio del Departamento de Comercio de los EE. UU., en agosto de 2000, el 78\% de los domicilios de este país con renta superior a USD 75 000/año tenían acceso a Internet, frente al 13\% de los domicilios con renta inferior a USD 15 000/ año. El $46 \%$ de los domicilios donde vivían blancos y el $57 \%$ donde vivían asiáticos estaban conectados a Internet, mientras que la cifra era del $23 \%$ para los domicilios donde vivían hispánicos y negros (7).

Los usuarios de Internet en los países de ALC todavía corresponden a una pequeña minoría de la población total, cerca del 4,7\%. Según el país, este promedio regional oscila entre más del $10 \%$ en

CUADRO 1. Usuarios de Internet por región del mundo, 2001

\begin{tabular}{lccc}
\hline \multicolumn{1}{c}{ Región } & $\begin{array}{c}\text { Usuarios de } \\
\text { Internet }\end{array}$ & $\begin{array}{c}\text { Usuarios } \\
\text { (por 100 habitantes) }\end{array}$ & $\begin{array}{c}\text { \% del total de usuarios } \\
\text { en el mundo }\end{array}$ \\
\hline EE. UU. y Canadá & 180680000 & 57,9 & 35,2 \\
Europa & 154630000 & 32,6 & 30,1 \\
Asia/Pacífico & 143990000 & 4,5 & 28,1 \\
América Latina y el Caribe & 24817000 & 4,7 & 4,8 \\
Medio Oriente & 4650000 & 1,6 & 0,9 \\
África & 4150000 & 0,6 & 0,8 \\
$\quad$ Total & 512917200 & & 100,0 \\
\hline
\end{tabular}

Fuente: NUA Internet Survey, agosto 2001. 
Argentina, Chile y Uruguay, y menos del 0,3\% en Guyana, Haití y República Dominicana. A pesar de que en términos absolutos la conectividad en la Región es pequeña comparada con la de otros países, su tasa de incremento anual (50\%) es la más alta del mundo, superior incluso a la de EE. UU., Europa y Asia. Se estima que en 2003 el número de usuarios en línea en ALC llegará a los 40 millones, representando el $7 \%$ de la población total (8).

La mayor parte de las redes de computación en ALC se han establecido en los últimos 8 años. Brasil y México (1989) fueron los primeros países en conectarse a servicios plenos interactivos de Internet. Desde el inicio del año 2000, varios países de ALC tienen más de mil servidores conectados a Internet. El número de servidores en Argentina, Brasil, Colombia, Costa Rica y Trinidad y Tobago se duplicó en el año 1999, y aumentó en un 90\% en Guatemala y Perú, mientras que en México el incremento fue de $259 \%$ en el mismo período.

Mientras el tren y la electricidad llevaron décadas para difundirse a los países en desarrollo, las TIC ya están avanzando en estos países a una velocidad dos veces mayor que en los desarrollados (9). De hecho, la propia revolución electrónica actualmente responsable de la "brecha digital", puede venir a ser responsable también de su disminución o desaparición. Para aumentar su red de comunicaciones, los países en desarrollo no tienen necesariamente que repetir las grandes inversiones hechas por los países desarrollados. En realidad, pueden evitar tecnologías intermedias, como los cables de cobre y los teléfonos analógicos, pasando a utilizar directamente las nuevas tecnologías sin cable que exigen menos inversiones y mantenimiento.

Los teléfonos celulares permiten extender la comunicación a sitios donde los cables de cobre tardarían muchos años en llegar. Nuevos productos de bajo costo, como los minicomputadores que se ajustan a la palma de la mano, permiten el acceso a Internet en cualquier lugar y no sería absurdo imaginar que en algunos años serán utilizados en las escuelas, como se hace hoy con las calculadoras manuales, que hasta hace poco también tenían precios prohibitivos.

Pese a la limitación del acceso a las nuevas tecnologías a un sector relativamente pequeño de la población latinoamericana, lo cual aumenta la situación de inequidad ya existente, es evidente que la tendencia será hacia una mayor disponibilidad de estos medios de comunicación. Es probable que en ALC se hagan efectivas las mismas tendencias observadas en EE. UU. y Canadá, donde se estima que el $54 \%$ de los usuarios de Internet la utilizan para obtener información o servicios relacionados con la salud (10). La mayor parte de los médicos estadounidenses se conectan a Internet diariamente y 55\% utilizan el correo electrónico para comunicarse con colegas y colaboradores (11). De manifestarse estas tendencias en el resto del hemisferio, para mediados del primer decenio del siglo XXI habrá más de 150 millones de personas (125 millones en EE. UU. y Canadá, y 25 millones en ALC) utilizando regularmente los medios cibernéticos para obtener servicios e información para el desarrollo de la salud, lo cual tendrá un impacto definitivo sobre las condiciones de salud y sus disparidades en la Región.

Sin embargo, la disminución de las desigualdades de acceso a las TIC seguirá dependiendo durante bastante tiempo de soluciones colectivas o públicas. Una única conexión a Internet puede ser compartida por muchos, dando, por ejemplo, a escuelas que nunca tuvieran una biblioteca un amplio acceso a las mejores bibliotecas del mundo y a sus alumnos la oportunidad de asistir a clases de los mejores profesores. En varios países de la región vienen proliferando servicios localizados en centros comunitarios y culturales, bibliotecas, escuelas, centros de salud y otros espacios públicos, denominados genéricamente telecentros, que ofrecen acceso a correo electrónico y conexión a Internet, especialmente en zonas marginales y remotas. Algunos telecentros ofertan incluso paquetes de educación a distancia, servicios de empleo y aplicaciones de telemedicina (12).

El aprovechamiento de estas y otras experiencias y oportunidades para superar el problema de las inequidades de información depende del establecimiento de políticas explícitamente diseñadas para tal fin. Estas políticas deben basarse en el reconocimiento de que la información, el conocimiento e Internet son bienes públicos y, por lo tanto, deben ser objeto de políticas públicas. Un bien público tiene básicamente dos características: la primera, que su consumo por parte de un individuo no impide que otro también lo consuma, o sea, no desaparece después de consumido, y en el caso de la información hasta se enriquece; la segunda, que es bastante difícil, si no imposible, excluir o impedir que determinado individuo disfrute de ese bien una vez que esté disponible.

Dadas estas dos características, las empresas privadas tienen pocos incentivos para invertir en la producción de conocimientos e informaciones, pues no pueden apropiarse totalmente de sus beneficios, a pesar de los intentos cada vez mayores de privatizar el acceso a estos bienes esencialmente públicos. En el momento de decidir dónde invertir, las empresas privadas tienden a privilegiar lo que les permite un retorno exclusivo a sí mismas y no lo que les permite a otros beneficiarse de su inversión. Por todo esto, el Estado debe cumplir un importante papel en la provisión de estos bienes, ya que de lo contrario habrá escasez de los mismos. 
En el caso de un bien público como Internet, el Estado debe actuar en varios frentes, como son la creación de la infraestructura básica de comunicaciones, el establecimiento de marcos reguladores, incluidos los estímulos para la participación del sector privado y, especialmente, la provisión de servicios que el mercado difícilmente proveerá, como son los de alto beneficio público y alto costo. Telemedicina y teleducación son un buen ejemplo de esto último, especialmente entre los pobres, donde los beneficios son grandes y las posibilidades de lucro limitadas. Con el avance de la globalización, en gran medida facilitada por las propias TIC, estos bienes públicos tienden cada vez más a transformarse en bienes públicos globales, lo que obliga que las políticas que estimulen su creación y distribución sean objeto de nuevos acuerdos institucionales de la comunidad internacional, incluyendo un nuevo papel de instituciones multilaterales tradicionales, como las agencias de Naciones Unidas, entre ellas la OPS y la Organización Mundial de la Salud (13).

La Organización de las Naciones Unidas está buscando responder a esta responsabilidad y un buen ejemplo es la reunión Cumbre del Milenio, realizada en septiembre de 2000 en Nueva York, en cuya declaración final los gobiernos participantes reconocen que el acceso a la información es un derecho básico y se comprometen a asegurar que los beneficios de las nuevas tecnologías de información y comunicación sean puestos a disposición de todos.

La BVS es otro destacado ejemplo de este nuevo papel de los organismos internacionales en actuación conjunta con las instituciones nacionales. Promoviendo el intercambio de este bien público global que es la información para el desarrollo social, la BVS se constituye en un poderoso instrumento para promover la acción colectiva con miras a la equidad.

\section{SYNOPSIS}

\section{Inequities in access to information and inequities in health}

This piece presents evidence that inequities in information are an important determinant of health inequities and that eliminating these inequities in access to information, especially by using new information and communication technologies (ICTs), could represent a significant advance in terms of guaranteeing the right to health for all. The piece reviews the most important international scientific research findings on the determinants of the health of populations, emphasizing the role of socioeconomic inequities and of deteriorating social capital as factors that worsen health conditions. It is noteworthy that Latin America has both socioeconomic inequities and major sectors of the population living in poverty. Among the fundamental strategies for overcoming the inequalities and the poverty are greater participation by the poor in civic life and the strengthening of social capital. The contribution that the new ICTs could make to these strategies is analyzed, and the Virtual Health Library (VHL) is discussed. Coordinated by the Latin American and Caribbean Center on Health Sciences Information (BIREME), the VHL is a contribution by the Pan American Health Organization that takes advantage of the potential of ICTs to democratize information and knowledge and consequently promote equity in health. The "digital gap" is discussed as something that can produce inequity itself and also increase other inequities, including ones in health. Prospects are discussed for overcoming this gap, emphasizing the role that governments and international organizations should play in order to expand access to the global public good that information for social development is.

\section{REFERENCIAS}

1. Wing S, Richardson D. Material living conditions and health in the United States, Canada and Western Europe: a review of recent literature. Washington, D.C.: Organización Panamericana de la Salud; 1999. (Serie Investigaciones en Salud Pública, No. 9).

2. Almeida Filho N. Desigualdades em saúde segundo condições de vida: análise da produção científica na América Latina e Caribe. Washington, D.C.: Organización Panamericana de la Salud; 1999. (Serie Investigaciones en Salud Pública, No. 19).

3. Wilkinson R. Unhealthy societies: the af flictions of inequality. New York: Routledge; 1997.

4. Evans RG, Barer ML, Marmor TR (eds.). Why are some people healthy and others not? Determinants of health of populations. New York: Aldine de Gruyter; 1994.

5. Kawachi I, Kennedy BP, Lochner K, Prothrow-Stith D. Social capital, income inequality, and mortality. Am J Public Health 1997;87: 1491-1498.

6. World Bank. World Development Report 2000/2001: Attacking poverty. New York: Oxford University Press; 2000.

7. US Department of Commerce. Falling through the Net: toward digital inclusion. Washington, D.C.: National Telecommunications and Information Administration; 2000. Disponible en: http:// www.ntia.doc.gov/ntiahome/fttn00/ contents00.html. Acceso el 30 abril 2002.

8. Hahn S. Case studies on development of the Internet in Latin America and the Caribbean. INET 2000, Internet Society Conference, Yokohama, Japan, 18-21 July, 2001. Disponible en http://www. isoc.org/isoc/conferences/ine/00/ cdproceedings/8d/8d_5.htm. Acceso el 30 abril 2002.

9. Woodall P. Untangling e-conomics: a survey of the new economy. The Economist 2000;356 (8189):S5.
10. Miller T, Reents S. The health care industry in transition: the online mandate to change. [Sitio en Internet] Cyber Dialogue. Disponible en: http://www. cyberdialogue.com/pdfs/wp/wp-cch1999-transition.pdf. Acceso el 30 abril 2002.

11. New data show Internet, Website and e-mail usage by physicians all increasing. [Sitio en Internet] Harris Interactive. Disponible en: http://www.harrisinteractive.com/news/allnewsbydate. asp? NewsID=237. Acceso el 30 abril 2002.

12. Gómez R. The hall of mirrors: the Internet in Latin America. Current History 2000;99 (634):72. Disponible en: http:// www.idrc.ca/pan/pubhall_e.htm. Acceso el 30 abril 2002.

13. Stiglitz JE. Knowledge as a global public goods. En: Kaul I, Grunberg I, Stern M (eds.). Global public goods. UNDP. New York: Oxford University Press; 1999. 